\title{
The data management plan - the common thread through the project. DMP consulting at the University of Vienna
}

\author{
Susanne Blumesberger \\ University of Vienna, Vienna University Library \\ Repositorymanagement PHAIDRA-Services, University of Vienna \\ Universitätsring 1, 1010 Wien
}

E-mail: susanne.blumesberger@univie.ac.at

This article will use the example of the University of Vienna to show how DMP consulting can succeed and that all parties involved benefit from it. Data Management Plans (DMPs), which are increasingly required by research funders and describe the life cycle of data within a research project, are not only important for the funders, but also for the repository operators and, above all, for the researchers themselves. The funders hope that this will result in the highest possible re-use rate of the data they have paid for, the repository operators know through DMPs what they need to focus their repository on, how much data of what size and complexity should be stored, and for the researchers it is a self-designed guide through the project. They define responsibilities for data protection, backup, etc. and have to think about legal and ethical issues, licensing, data description, necessary technical implementations, archiving and storage options. If the questions are clarified in the DMP's, one also receives an overview of which data can be further used under which conditions. Optimally completed data management plans also make the data fit for further use in a Citizen Science project and possibly for further improvement. Data management plans are therefore useful even where they are not yet mandatory, because they structure the workflow and help to plan the necessary resources. Data management plans, which should be living documents, are also usefully linked to other services such as repositories, DOI allocation, ORCID, etc. In the best case, the DMP also shows that there are still no appropriate solutions for some requirements, for example for the long-term accessibility of databases. 


\section{Introduction}

For many researchers, the data management plan is initially an additional hurdle on the way to a third-party funded project. They are confronted with a number of questions that they have not yet dealt with and also do not know exactly what the funding body wants to know. In addition to questions about administration, for example, they should also specify what data will be produced, how large the amount of data will be, how it will be documented, what quality assurance is planned, who will have access to the data and, above all, what will happen to the data used or created after the project ends. In addition, there are questions about whether there could be legal or ethical problems in opening up the data. Successfully addressing these questions is the foundation for successfully conducting a Citizen Science project. At this stage, when data management plans have not been mandatory for too long by some funding agencies, scientists often feel overwhelmed. For example, as of Jan. 1, 2019, the Austrian Science Fund requires a DMP after the project has been approved.

Admittedly, it takes time to get to grips with these requirements, but it is always rewarding. (1) To successfully conduct and complete a scientific project requires at least a minimal version of project management to be able to plan processes and costs. It is the same with data management. In order to archive data securely and to handle them according to the FAIR principles (2) i.e. to make them findable, accessible, interoperable and reusable, some decisions have to be made in advance. Apart from that, it is helpful to define who is responsible for which tasks in which project phases.

\section{The data management plan as a tool to open data}

As a research support office (3) at the large and heterogeneous University of Vienna, we deal with very different data. We advise researchers from different disciplines such as physics, humanities and social sciences, who want to make their data available to the entire world as quickly as possible to scientists who want or need to keep their data closed for various reasons. The fact that data is not made available very often does not mean that the will to do so is not there, but that people are well aware of the hurdles and act responsibly. These include, for example, ethical concerns, legal restrictions, but also considerations as to whether one really wants to make the data freely available to other researchers while the project is still in progress in a highly competitive research area.

Even if the data itself, or not all of the data, is immediately freely available, this does not mean that one does not adhere to the FAIR Data Principles, but rather that one handles one's own research output prudently and possibly releases the data gradually. The transparency so often demanded is also given if one initially only opens the descriptions, i.e. the metadata, even if the data itself cannot be offered openly for good reason, or only to selected persons. The transparency required by the FAIR principles also means making one's own research process visible. In principle, however, our goal is to encourage researchers to open their data as much as possible. After all, offering open data also means being visible to the public, an essential factor in today's research landscape. As an emerging field CS can benefit from the use of DMP in multiple ways such as collaborative, cross-disciplinary research, possibly involving the public. Citizen science projects are very popular and also successful. (4) 


\section{Consulting - many paths lead to the goal}

The consulting activities in the area of research data management at the Vienna (5) University Library have developed primarily from the repository management. When the university-wide repository Phaidra went live in 2008, our core task was primarily to keep the system running, and help interested researchers archive their texts, images, videos, and audio files there. The openness of the system, the interfaces that were available from the beginning, the permanent signatures, and the machine readability of the data already met the requirements of the FAIR principles, which were published only later. Parallel to the technical development of the system, the consulting services were expanded. It was no longer just about the pure upload process, but more generally about research data management. When the FWF required data management plans (6) for approved projects on Jan. 1, 2019, the demand for advice and support from researchers who needed to complete such a plan increased. We take a multi-pronged approach to training and workshops. Through Staff Development, we offer training on uploading to Phaidra, on data management, and separately on data management plans, in addition to open access consulting. In addition, we also offer one-on-one meetings with project managers and staff, by phone, in person, or digitally. This is complemented by a website (7) that provides a wealth of information on data management and data management plans and is constantly updated, as well as guidelines and further reading. Researchers from various disciplines come to us with different inquiries, very often they are legal uncertainties, but also questions about the technical feasibility of the projects. We try to respond individually to the projects and, if necessary, to use customized solutions in addition to standard products.

\section{Data management plans - a win-win for everyone}

Completing a DMP should not only be seen as a burden, but also as an opportunity. (8) Thinking about one's own project naturally has the advantage for the researchers themselves that there is clarity about the process, the costs and any additional services that may be required, be it legal or technical advice. Perhaps one would not have thought of legal problems at all, all the better if uncertainties in this regard have to be clarified before the start of the project. Some funding bodies, such as the EU, require at least two updates of the data management plan, once in the middle of the project and once at the end. This means that researchers always have an up-to-date plan, which should be accessible to all and thus reflect the status quo of the project. The funding agencies benefit from DMP's because they can use them to control that data that is allowed to be open is also made public, that it is stored securely in a trusted repository and is permanently available. By requiring reusable data, they hope to save costs, and existing resources are used transparently and optimally.

Last but not least, it gives them a good overview of the projects. For repository managers DMP's are also helpful, because in case of consultations they get insight into the respective research projects and thus learn about special requirements. This makes it easier to estimate the time and resources required.

Of course, data management plans are also an asset for Citizen Science projects. Data and metadata are available in an optimal quality, the data are provided with permanent signatures and licenses, and furthermore, legal issues are clarified.

From this point of view, data management plans are very helpful for all parties involved, because they make research FAIR, through permanent identifiers, interfaces, machine readability, multilingualism, standardized metadata and vocabularies, legal security, and last but not least, secure archiving. 


\section{References}

(1) Mayer, Katja: Who is afraid of the Data Management Plan? Exploration of the DMPs as useful tools in the research process. https://phaidra.univie.ac.at/o:1169818 (25.3.2021)

(2) The Data FAIR Principles. https://www.force11.org/group/fairgroup/fairprinciples (25.3.2021)

(3) https://datamanagement.univie.ac.at/en/ (19.3.2021)

(4) One example is the Vorarlberg Provincial Repository, which uses Citizen Science projects to improve the descriptions of its numerous images. https://pid.volare.vorarlberg.at/ (31.3.2021)

(5) See also: https://phaidra.univie.ac.at/o:1099950 (19.3.2021)

(6) https://m.fwf.ac.at/en/research-funding/open-access-policy/research-data-management (19.3.2021)

(7) https://datamanagement.univie.ac.at/en/research-data-management/ (19.3.2021)

(8) Der Datenmanagementplan - der rote Faden durch das Projekt. DMP-Beratung an der Universität Wien. https://phaidra.univie.ac.at/o:1099950 (19.3.2021) 